\title{
Neutron diffraction study of nuclear magnetic ordered phases and domains in lithium hydride
}

\author{
Y. Roinel, G. L. Bacchella, O. Avenel, V. Bouffard, M. Pinot, P. Roubeau, P. Meriel and M. Goldman \\ Service de Physique du Solide et de Résonance Magnétique, \\ Centre d'Etudes Nucléaires de Saclay, B.P. no 2, 91190 Gif sur Yvette, France
}

(Reçu le 27 novembre 1979, accepté le 4 janvier 1980)

\begin{abstract}
Résumé. - On décrit la détection par diffraction de neutrons de deux nouvelles phases ordonnées dans LiH, produites par désaimantation adiabatique nucléaire dans un champ élevé parallèle à un axe cristallin [110]. Les phases sont : antiferromagnétique à température positive, et ferromagnétique à température négative.

Un détecteur de neutrons bidimensionnel révèle que les domaines sont anisotropes, sous la forme de disques perpendiculaires au champ, aussi bien pour la phase ferromagnétique (conformément aux prévisions) que pour la phase antiferromagnétique. Des valeurs approchées sont données pour les dimensions longitudinales et transverses des domaines.
\end{abstract}

\begin{abstract}
We describe the detection by neutron diffraction of two new ordered phases in LiH, produced by nuclear adiabatic demagnetization in a high field parallel to a [110] crystalline axis. The phases are antiferromagnetic at positive temperature and ferromagnetic at negative temperature.

A 2-dimensional neutron detector reveals that the domains are anisotropic, in the form of pancakes perpendicular to the field, both in the ferromagnet as expected, and in an antiferromagnet. Approximate values are derived for the longitudinal and transverse dimensions of the domains.
\end{abstract}

We have described in a preceding letter the detection by neutron diffraction of two antiferromagnetic structures in lithium hydride [1]. In the present letter, we report the evidence obtained by neutron diffraction for the existence of two new ordered phases in this compound : an antiferromagnetic and a ferromagnetic phase, as well as a preliminary study of the shape and size of the ordered domains through the use of twodimensional neutron counter.

Nuclear magnetic ordering has been studied for several years, essentially by NMR methods, at the laboratory of Nuclear Magnetism at Saclay. The principles and methods for the production and study of nuclear dipolar ordered phases in dielectric crystals have been described in several articles [2, 3, 4].

The cooling of the spins below the critical temperature $T_{\mathrm{c}}$, in the $\mu \mathrm{K}$ range, is achieved by a two-step process : dynamic polarization, followed by adiabatic demagnetization in the rotating frame. The final Hamiltonian is the truncated dipolar Hamiltonian $\mathcal{H}_{\mathrm{D}}^{\prime}$. The nature of the ordered phase depends both on the orientation of the external field with respect to the crystalline axes (because the Hamiltonian $\mathcal{H}_{\mathrm{D}}^{\prime}$ depends on this orientation), and on the sign of the spin temperature.
The neutron diffraction study of magnetic ordering is possible because of the large spin-dependent neutron-proton scattering amplitude, originating from the strong interaction between neutrons and protons.

The apparatus was briefly described in references [1, 7 and 8]. The sample, cooled to about $50 \mathrm{mK}$ is in a vertical field of $6.5 \mathrm{~T}$, and the neutron diffraction plane is horizontal. The neutron wavelength is $1.13 \AA$.

1. Detection of new ordered structures. - The crystal structure of $\mathrm{LiH}$ is of the $\mathrm{NaCl}$ type : two interpenetrating fcc lattices. In reference [1] we have described the neutron-diffraction study of the phases produced by demagnetization when the field $H$ is parallel to a [001] axis. The structures at $T>0$ and $T<0$ are antiferromagnetic and both give rise to the same 110 neutron reflexion. That they are different is proved by the very different values of critical entropy.

In the present letter we describe the detection of the ordered phases produced by demagnetization in high field when the field $H$ is parallel to a [011] axis. The structures predicted for that case by a local Weissfield approximation [2] are shown in figure 1. At positive temperature it is antiferromagnetic, and at negative temperature it is ferromagnetic with domains 

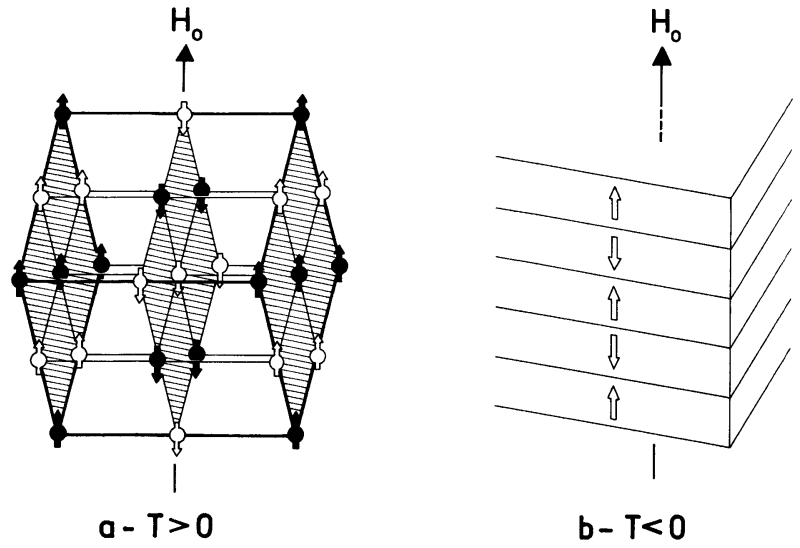

Fig. 1. - Nuclear ordered structures in $\mathrm{LiH}$ for $H / /$ [011] at positive and negative temperatures. White : ${ }^{1} \mathrm{H}$ spins; black : ${ }^{7} \mathrm{Li}$ spins. The hatched planes in $a$ ) are those on which the neutrons are diffracted. In each domain of $b$ ) the ${ }^{1} \mathrm{H}$ and ${ }^{7} \mathrm{Li}$ spins are pointing in the same direction.

in the form of thin slices perpendicular to the field.

The existence of the antiferromagnet at $T>0$ is detected by the 100 neutron diffraction line, and that of the ferromagnet at $T<0$ by a modification of the 200 crystalline neutron diffraction line.

The rocking curves observed for both sign of spin temperature are shown in figure 2. The 100 line at $T>0$ is a characteristic proof that the antiferromagnet has indeed the theoretical structure of figure $1 a$. At $T<0$, the occurrence of ferromagnetism with domains shows up by a broad line superimposed on the narrow crystalline line. Its width is attributed to the finite size of the domains, as discussed below.

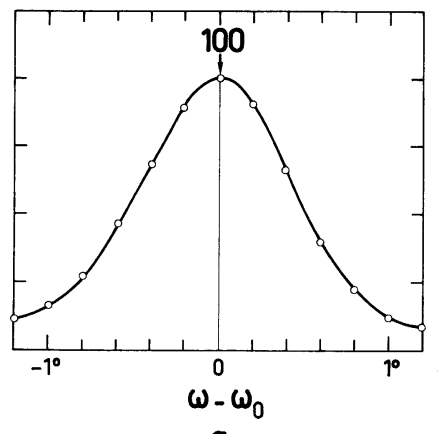

a

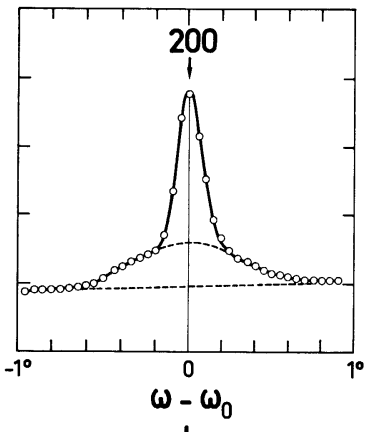

b
Fig. 2. - Neutron diffraction rocking curves in the ordered structures of figure $1: a$ ) Reflexion 100 at $T>0$. Continuous background substracted. $b$ ) Reflexion 200 at $T<0$. Continuous background not substracted.

2. Domain sizes and shapes. - The existence of domains in an ordered system is characterized by average polarizations of individual nuclei of the form :

$$
p_{i}=g_{i} \exp \left[i \mathbf{K}_{0} \cdot \mathbf{R}_{i}\right]
$$

where $\mathbf{K}_{0}$ is the wave vector corresponding to the ordering (ferro or antiferromagnetic) and $g_{i}$ varies from +1 to -1 in successive domains. By a Fourier transform of the $g_{i}$, eq. (1) becomes :

$$
p_{i}=\sum_{q} A(\mathbf{q}) \exp \left[i\left(\mathbf{K}_{0}+\mathbf{q}\right) \cdot \mathbf{R}_{i}\right] .
$$

The existence of domains causes the variation of nuclear polarization to be a sum of periodical variations with a spread of wave vectors around $\mathbf{K}_{0}$, and a corresponding variation for the neutron scattering amplitude. The width $\Delta q$ of this wave vector distribution along a given direction is of the order of the inverse domain size in this direction. This spread of wave vectors give rise both to an angular spread of the neutron diffracted beam around the Bragg direction, and to a broadening of the rocking curve.

A complete analysis of the relationship between domain distribution and neutron diffraction pattern will be given in a forthcoming article. Anticipating on this calculation, it can be shown that the ideal rocking curve is, the Fourier transform of the autocorrelation function $\varphi$ of $g_{i}=g\left(R_{i}\right)$ in the direction of the diffracted beam :

$$
\varphi(\mathbf{r})=\sum_{i} g\left(\mathbf{R}_{i}^{\prime}\right) g\left(\mathbf{R}_{i}+\mathbf{r}\right) .
$$

The width of the diffraction lines corresponding to ordering being much broader than those of the crystalline diffraction lines $\left(0.6\right.$ to $1^{\circ}$ versus $\left.0.2^{\circ}\right)$, the rocking curves can be used, after correcting for the effect of neutron beam and crystal imperfections, to derive the average domain size along the diffracted beam. The results obtained for three different ordered phases in $\mathrm{LiH}$ are the following :

$$
\begin{aligned}
& H / /[001], T<0, \mathrm{AF}: \bar{r} \simeq 160 \text { to } 200 \AA \\
& H / /[011], T>0, \mathrm{AF}: \bar{r} \simeq 160 \text { to } 200 \AA \\
& H / /[011], T<0, \text { Ferro }: \bar{r} \simeq 100 \text { to } 150 \AA .
\end{aligned}
$$

These studies on domains were extended by the use of a 2-dimensional neutron multidetector. The prototype apparatus was kindly lent to us by the LETI of Grenoble. The detailed study of the diffracted beam angular spread, allowed by this kind of detector, is potentially capable of yielding the domain size distribution in all directions. We have performed only preliminary measurements. An example of the results is shown in figure 3. The magnetic field is along the [011] axis and the crystal is oriented at the exact Bragg angle of the 200 reflexion. On this figure are drawn the lines of equal neutron intensity :

a) before the nuclear demagnetization, that is in the absence of dipolar ordering;

b) after demagnetization at negative temperature.

The ordered phase is the ferromagnet with domains of figure $1 b$. The enormous vertical elongation of the neutron blot shows that the domain thickness parallel to the field is much less than their transverse dimensions. This result proves that the shape of the domains, 


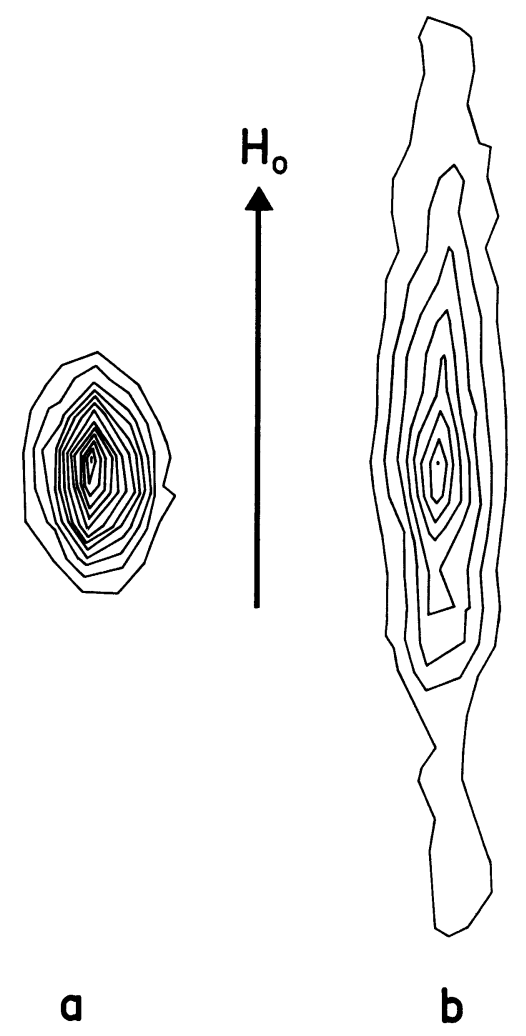

Fig. 3. - Lines of equal intensity of the neutron beam for the 200 reflexion at the centre of the rocking curve. a) Before demagnetization. No dipolar order. The intensity is enhanced by the large nuclear polarization. b) After demagnetization at $T<0$. Ferromagnetic structure $1 \mathrm{~b}$.

as well as their orientation is indeed as expected theoretically.

The 2-dimensional counter was also used for observing the antiferromagnetic reflexion 110 , in the AF phase corresponding to $H / /$ [001], $T<0$. There again the neutron blot is elongated, revealing anisotropic domains in the form of pancakes perpendicular to the field. The reason for this anisotropy is not understood at present.

The following very crude estimates are derived for the domain thickness in these two phases :

$$
\begin{aligned}
& H / /[001], T<0, \mathrm{AF}: \bar{d} \sim 40 \AA \\
& H / /[011], T<0, \text { Ferro }: \bar{d} \sim 20 \AA .
\end{aligned}
$$

These values are comparable with the average distance between the paramagnetic F-centres, $\sim 40 \AA$, which seems to indicate the importance of the paramagnetic impurities for the initiation of the domains.

3. Conclusion. - Four different nuclear ordered phases have already been observed by neutron diffraction in one substance : $\mathrm{LiH}$, two of them described in reference [1] and two in the present letter. These observations provide a striking illustration of one of the most noticeable features of nuclear dipolar ordering in high field : the possibility of producing different orderings by changing the field orientation or the sign of the temperature.

The 2-dimensional neutron detector proves to be a unique tool for the investigation of anisotropic domains. The size of the domains turn out to be large enough to allow one to speak of long range order, but small enough to be revealed and studied by neutron diffraction.

Acknowledgments. - This work has greatly benefited from the stimulus and interest of Professor Abragam.

\section{References}

[1] Roinel, Y., Bouffard, V., Bacchella, G. L., Pinot, M., Meriel, P., Roubeau, P., Avenel, O., Goldman, M. and Abragam, A., Phys. Rev. Lett. 41 (1978) 1572.

[2] Goldman, M., Phys. Rep. 32C (1977) 1.

[3] Roinel, Y., Bouffard, V. and Roubeau, P., J. Physique 39 (1978) 1097.

[4] Abragam, A., Bouffard, V., Goldman, M. and Roinel, Y., J. Physique Colloq. 39 (1978) C6-1436.
[5] Abragam, A. and Goldman, M., Rep. Prog. Phys. 41 (1978) 395.

[6] Goldman, M., Spin temperature and nuclear magnetic resonance in solids (Oxford University Press) 1970, Chap. 2.

[7] Bacchella, G. L., Meriel, P. and Pinot, M., J. Physique Colloq. 39 (1978) C6-810.

[8] Roubeau, P., Roinel, Y., Bouffard, V. and Avenel, O., J. Physique Colloq. 39 (1978) C6-1146. 